\title{
Superior Petrosal Sinus: Hemodynamic Features in Normal and Cavernous Sinus Dural Arteriovenous Fistulas
}

\author{
R. Shimada, H. Kiyosue, S. Tanoue, H. Mori, and T. Abe
}

\begin{abstract}
BACKGROUND AND PURPOSE: Normal hemodynamic features of the superior petrosal sinus and their relationships to the SPS drainage from cavernous sinus dural arteriovenous fistulas are not well known. We investigated normal hemodynamic features of the SPS on cerebral angiography as well as the frequency and types of the SPS drainage from CSDAVFs.
\end{abstract}

MATERIALS AND METHODS: We evaluated 119 patients who underwent cerebral angiography by focusing on visualization and hemodynamic status of the SPS. We also reviewed selective angiography in 25 consecutive patients with CSDAVFs; we were especially interested in the presence of drainage routes through the SPS from CSDAVFs.

RESULTS: In 119 patients (238 sides), the SPS was segmentally (anterior segment, 37 sides; posterior segment, 82 sides) or totally (116 sides) demonstrated. It was demonstrated on carotid angiography in 11 sides (4.6\%), receiving blood from the basal vein of Rosenthal or sphenopetrosal sinus, and on vertebral angiography in 235 sides (98.7\%), receiving blood from the petrosal vein. No SPSs were demonstrated with venous drainage from the cavernous sinus. SPS drainage was found in 7 of 25 patients (28\%) with CSDAVFs. CSDAVFs drained through the anterior segment of SPS into the petrosal vein without draining to the posterior segment in 3 of 7 patients (12\%).

CONCLUSIONS: The SPS normally works as the drainage route receiving blood from the anterior cerebellar and brain stem venous systems. The variation of hemodynamic features would be related to the relatively lower frequency and 2 different types of SPS drainage from CSDAVFs.

ABBREVIATIONS: $C A G=$ carotid angiography; $C S D A V F=$ cavernous sinus dural arteriovenous fistula; $S P S=$ superior petrosal sinus; $V A G=$ vertebral angiography

$\mathrm{T}$ he SPS is well known as a dural sinus that communicates with the cavernous sinus anteriorly and with the transverse sinus posteriorly. It runs over the trigeminal nerve in the lateral margin of the tentorium and in the superior petrosal sulcus of the temporal bone. In some textbooks, the SPS is described as a dural sinus that receives blood from the cavernous sinus and drains into the transverse sinus. ${ }^{1,2}$ Normal hemodynamic features of the SPS have not been reported, however, and the SPS is not frequently demonstrated at venous phase on internal carotid angiography in our clinical practice.

Furthermore, cavernous sinus dural arteriovenous fistulas less frequently drain via the SPS to the transverse sinus. ${ }^{3-5}$ In this study, we investigated the normal hemodynamic feature of the

Received March 7, 2012; accepted after revision June 13.

From the Department of Radiology (R.S., H.K., S.T., H.M.), Oita University Faculty of Medicine, Oita, Japan; and Department of Radiology (T.A.), Kurume University School of Medicine, Kurume City, Fukuoka, Japan.

Please address correspondence to Hiro Kiyosue, MD, Department of Radiology, Oita University Faculty of Medicine, 1-1 Idaigaoka, Hasama-machi, Yufu-shi, Oita, 879-5593, Japan; e-mail: hkiyosue@oita-u.ac.jp

http://dx.doi.org/10.3174/ajnr.A3252
SPS on cerebral angiography as well as the frequency and types of SPS drainage from CSDAVFs.

\section{MATERIALS AND METHODS}

For evaluating normal hemodynamic features of the SPS, we retrospectively reviewed 119 consecutive patients who underwent cerebral angiography between May 2010 and October 2011 at our institution. Patients who had lesions affecting cerebral venous drainage were excluded from this study. Patient age ranged from 12 to 84 years (mean age, 56.6 years), and there were 56 males and 63 females. Selective digital subtraction angiography of the bilateral common/internal carotid arteries and the vertebral arteries was performed in all patients without breath-holding by using biplane angiography equipment (Infinix Celeve-i INFX-8000V; Toshiba Medical Systems, Tokyo, Japan). Two experienced neuroradiologists evaluated the images carefully by focusing on visualization of the SPS and its hemodynamic status.

We also reviewed selective angiography from 25 consecutive patients with CSDAVFs in whom angiography was performed between September 2003 and January 2011 at our institution. There were 4 
men and 21 women, ranging in age from 55 to 81 years (mean age, 69.7 years). Selective angiography of bilateral external carotid arteries, internal carotid arteries, and vertebral arteries were reviewed, and special attention was paid to the presence of drainage routes through the SPS from CSDAVFs. We also evaluated the normal cerebral venous drainage via the SPS of the affected side in the patients with CSDAVFs.

\section{RESULTS}

\section{Normal Hemodynamic Features of the SPS}

In 119 patients, the SPSs were partially or totally demonstrated on either carotid angiography or vertebral angiography in 235 of 238 sides. The SPS was not found in 3 sides (Fig 1) on CAG or VAG. The hemodynamic status of the SPS in the patients with normal cerebral venous circulation is summarized in Tables 1 and 2. On CAG, the SPS was demonstrated in 11 sides (4.6\%). In 8 sides, the SPS was partially demonstrated to receive blood from the basal vein of Rosenthal via the anastomosis of the lateral mesencephalic vein and the petrosal vein, which included in 6 sides the anterior segment of the SPS with venous drainage from the petrosal vein to the cavernous sinus and in 2 sides the posterior segment with venous drainage from the petrosal vein to the transverse sinus (Fig $2 A-D$ ). In 2 sides, the superficial middle cerebral vein was found to terminate into the anterior segment of sphenopetrosal sinus (Fig 2E, $-F$ ). In the remaining side, the whole SPS was demonstrated with the blood flow from the transverse sinus into the cavernous sinus via the SPS (Fig 3). On VAG, the SPS was demonstrated to receive blood from the petrosal vein in 235 sides (98.7\%). Among these, the whole SPS was demonstrated in 116 sides (48.7\%) and partially demonstrated in 119 sides (50\%); of the 119 partial demonstrations, it appeared at the posterior segment in 82 sides (34.5\%) and at the anterior segment in 37 sides (15.5\%) (Figs 4 and 5). Additional findings included variations of the SPS, and duplication of the SPS and disconnection between the anterior segment (5 sides [2\%]) and the posterior segments (6 sides [2.5\%]) of the SPS was observed (Fig 6). Venous drainage from the cavernous sinus on both CAG and VAG was not demonstrated.

\section{Patients with CSDAVFs}

SPS drainage was found in 7 (28\%) of 25 patients with CSDAVFs. Table 3 summarizes the hemodynamic status of the SPS in the 7 patients with SPS drainage from the CSDAVFs. Among these 7patients, the CSDAVFs drained through the whole SPS into the transverse sinus in 3 patients (12\%) (Fig 7) and through the anterior segment of the SPS into the tributes of the petrosal vein without draining 

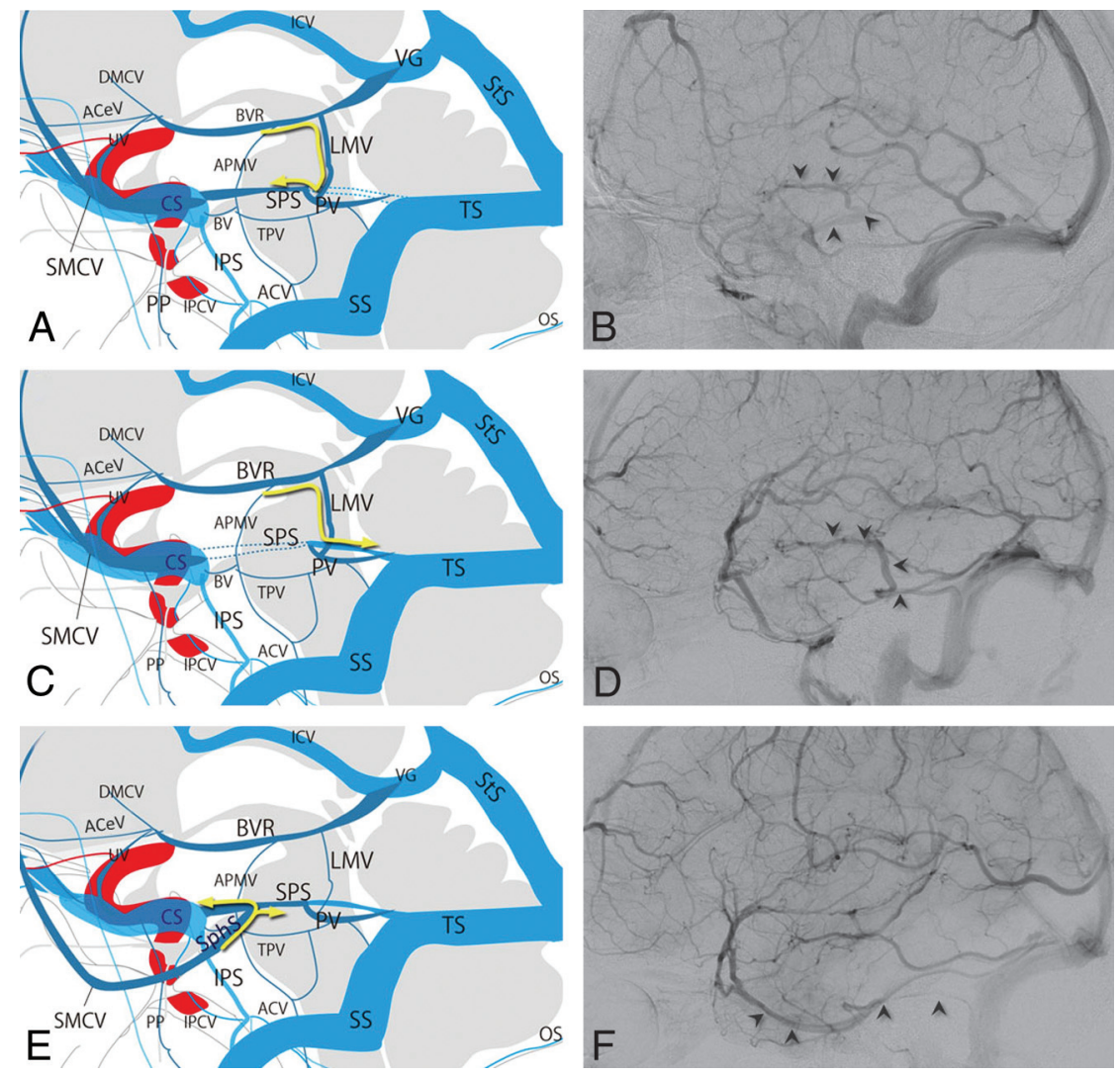

FIG 2. Types of demonstration of normal SPS drainage on carotid angiography. $A, C$, and $E$, Schematic drawings of SPS demonstration on carotid angiography. $B, D$, and $F$, Lateral views of carotid angiography in the venous phase. Schematic drawing $(A)$ and lateral view $(B)$ of carotid angiography demonstrate the SPS drainage route (arrowheads) from the basal vein of Rosenthal (BVR) via the lateral mesencephalic vein (LMV), the petrosal vein (PV), and the anterior segment of the SPS into the cavernous sinus (CS). Schematic drawing $(C)$ and lateral view (D) of carotid angiography demonstrate the SPS drainage route (arrowheads) from the BVR via the LMV, the PV, and the posterior segment of the SPS into the transverse sinus. Schematic drawing $(E)$ and lateral view $(F)$ of carotid angiography demonstrate the SPS drainage route (arrowheads) from the superficial middle cerebral vein (SMCV) via the sphenopetrosal sinus (primitive tentorial sinus) and both segments of the SPS. ACeV indicates anterior cerebral vein; ACV, anterior condylar vein; APMV, anterior pontomesencephalic vein; BV, bridging vein; DMCV, deep middle cerebral vein; ICV, internal cerebral vein; IPCV, inferior petroclival vein; IPS, inferior petrosal sinus; OS, occipital sinus; PP, pterygoid plexus; SphS, sphenopetrosal sinus; SS, sigmoid sinus; StS, straight sinus; TPV, transverse pontine vein; TS, transverse sinus; UV, uncal vein; VG, vein of Galen.
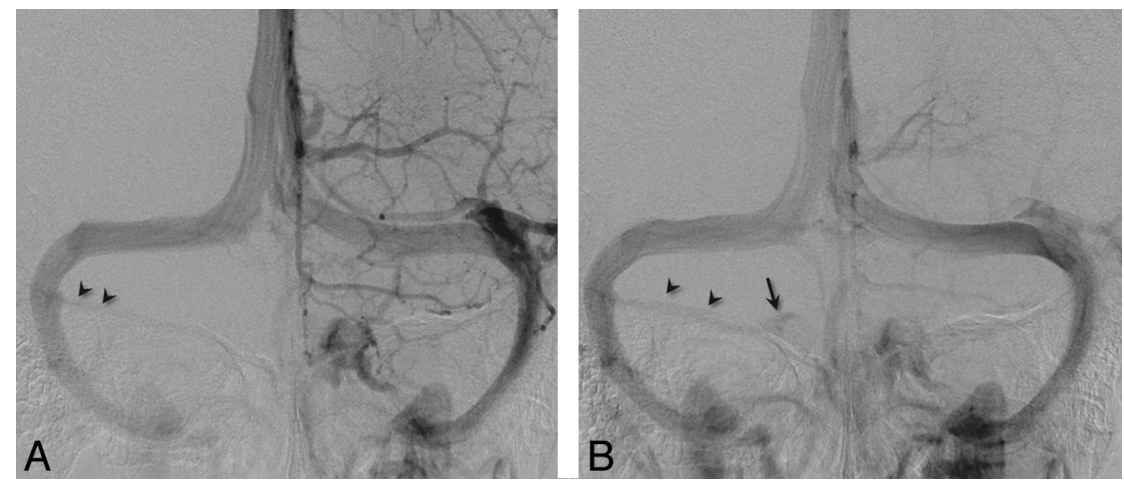

FIG 3. Demonstration of the SPS on carotid angiography with the blood flow from the transverse sinus to the CS. Sequential images of frontal views of the left carotid angiogram show that the blood flow from the right transverse sinus partially fills the SPS (arrowheads) and continues to the right cavernous sinus (arrow).

into the posterior segment of the SPS in 3 patients (12\%) (Fig 8). In the remaining patient, the CSDAVF drained through the anterior segment of SPS into the superficial middle cerebral vein (sphenopetrosal sinus), in which duplication of the SPS was also found (Fig 9).
In 18 CSDAVFs without SPS drainage, the SPS was partially observed at the posterior segment alone in 13 patients and at the anterior segment alone in 4 patients. The SPS was not found in 1 patient in whom other cerebellar veins were well developed. 

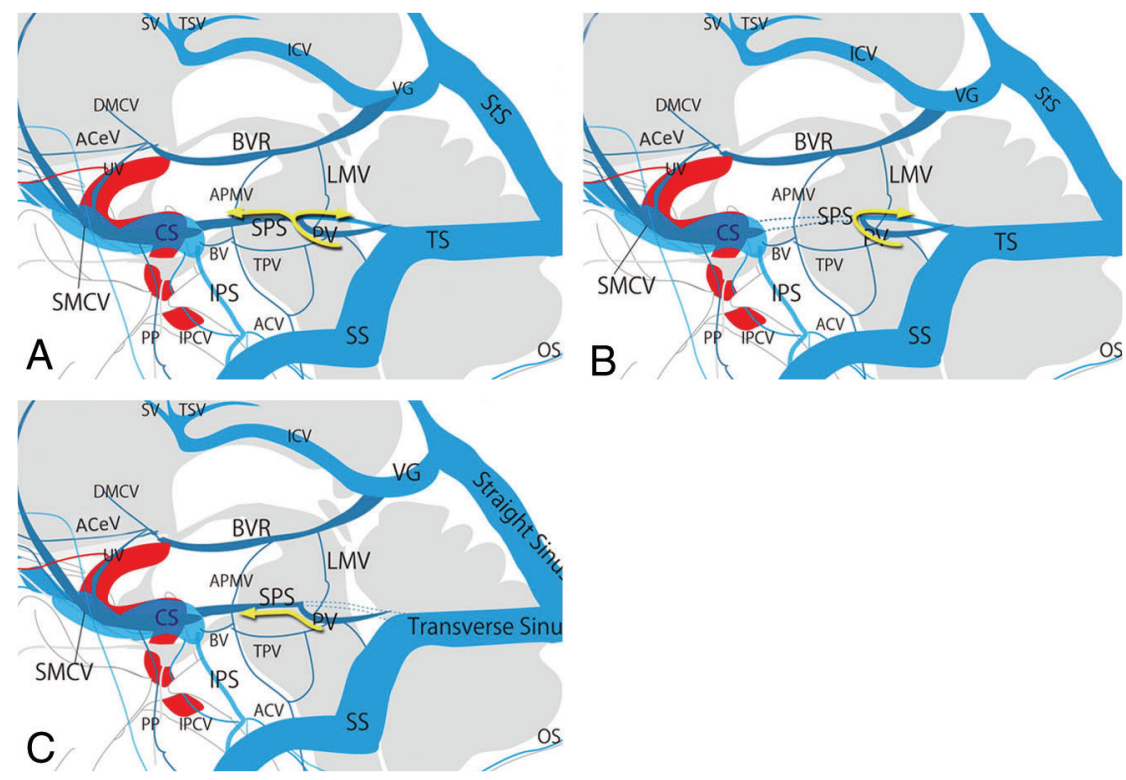

FIG 4. Schematic drawing of the hemodynamic types of the SPS based on vertebral angiography. A, Drainage from the petrosal vein (PV) into both the anterior and the posterior segment of the SPS (yellow arrow). B, Drainage from the PV into the posterior segment of the SPS to the transverse sinus (TS) alone (yellow arrow). C, Drainage from the PV into the anterior segment of the SPS to the cavernous sinus (CS) alone (yellow arrow). ACeV indicates anterior cerebral vein; ACV, anterior condylar vein; APMV, anterior pontomesencephalic vein; BV, bridging vein; BVR, basal vein of Rosenthal; DMCV, deep middle cerebral vein; ICV, internal cerebral vein; IPCV, inferior petroclival vein; IPS, inferior petrosal sinus; LMV, lateral mesencephalic vein; OS, occipital sinus; PP, pterygoid plexus; SMCV, superficial middle cerebral vein; SS, sigmoid sinus; StS, straight sinus; TPV, transverse pontine vein; TS, transverse sinus; UV, uncal vein; VG, vein of Galen.
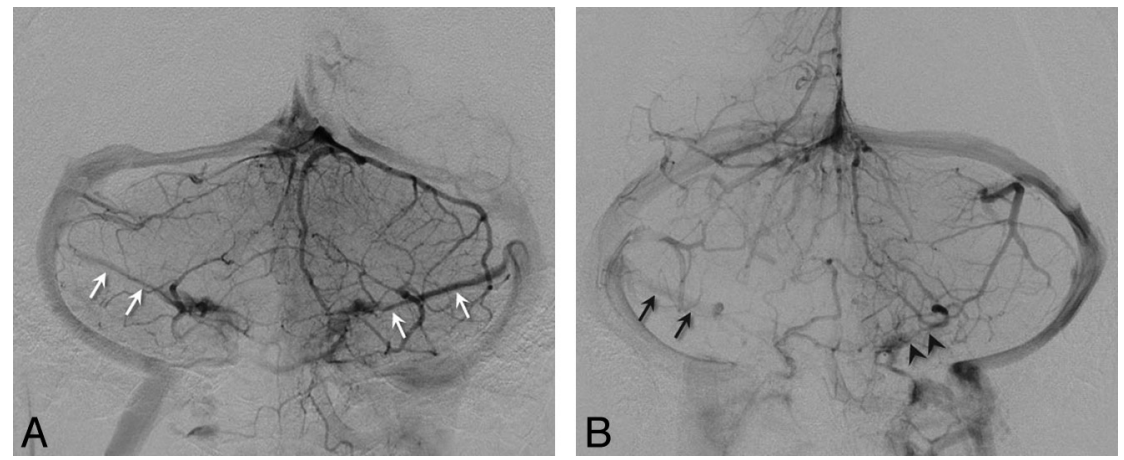

FIG 5. Frontal view of the left vertebral angiography at venous phase. $A$, The whole SPS was demonstrated bilaterally (white arrows). $B$, The right SPS was demonstrated on the anterior segment alone (black arrows). The left SPS was demonstrated on the anterior segment alone (arrowheads).
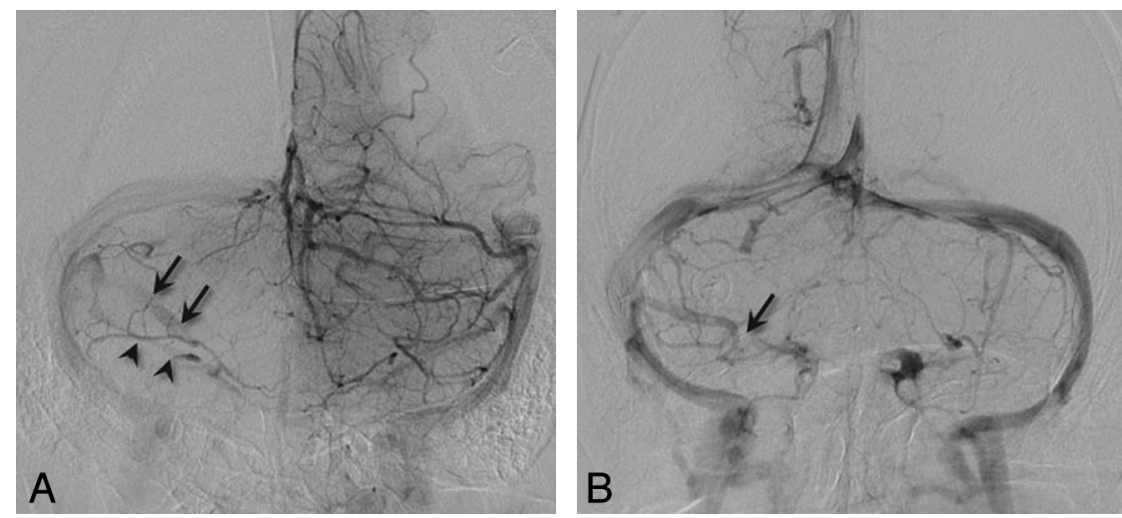

FIG 6. Frontal views of the vertebral angiogram show variations of the SPS. A, The right SPS reveals duplication of the superior segment (arrows) and inferior segment (arrowheads). The inferior segment is connected to the cavernous sinus. $B$, The right SPS reveals disconnection of the anterior and posterior segments (arrow). Both segments drain individually into the transverse sinus and the cavernous sinus. 
Table 3: Patterns of SPS drainage in the cases of CSDAVF $(n=7)$ Drainage Patterns Number of Cases

$\mathrm{CS} \rightarrow$ SPS $\rightarrow$ SS
$\mathrm{CS} \rightarrow$ SPS $\rightarrow$ PV
$\mathrm{CS} \rightarrow$ SPS $\rightarrow$ SphS

Note:-CS indicates cavernous sinus; PV, petrosal vein; SphS, sphenopetrosal sinus; SPS, superior petrosal sinus; SS, sigmoid sinus.

\section{DISCUSSION}

Embryologic Development and Hemodynamic Features of SPS

In the development of the cerebral venous system, the pro-otic sinus participates in forming the cavernous sinus and foramen ovale venous plexus (pterygoid plexus) and receives blood flow from the superior ophthalmic vein. After developing to the
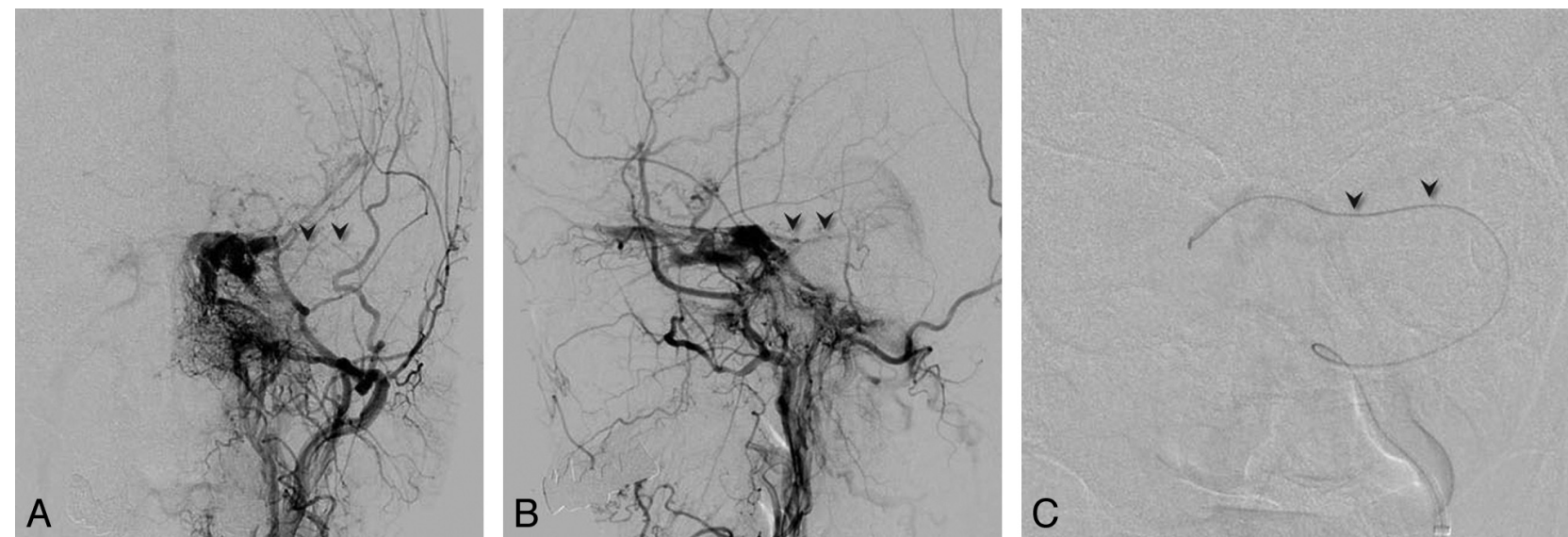

FIG 7. CSDAVF with SPS drainage. Frontal $(A)$ and lateral $(B)$ views of the left external carotid angiogram show the AVF draining into the left SPS (arrowheads). Additional drainage routes of the superior ophthalmic vein, the inferior petrosal sinus, the intercavernous sinus, and the superficial middle cerebral vein are also noted. C, Microcatheter is inserted into the right cavernous sinus via the left SPS (arrowheads) for the transvenous embolization.
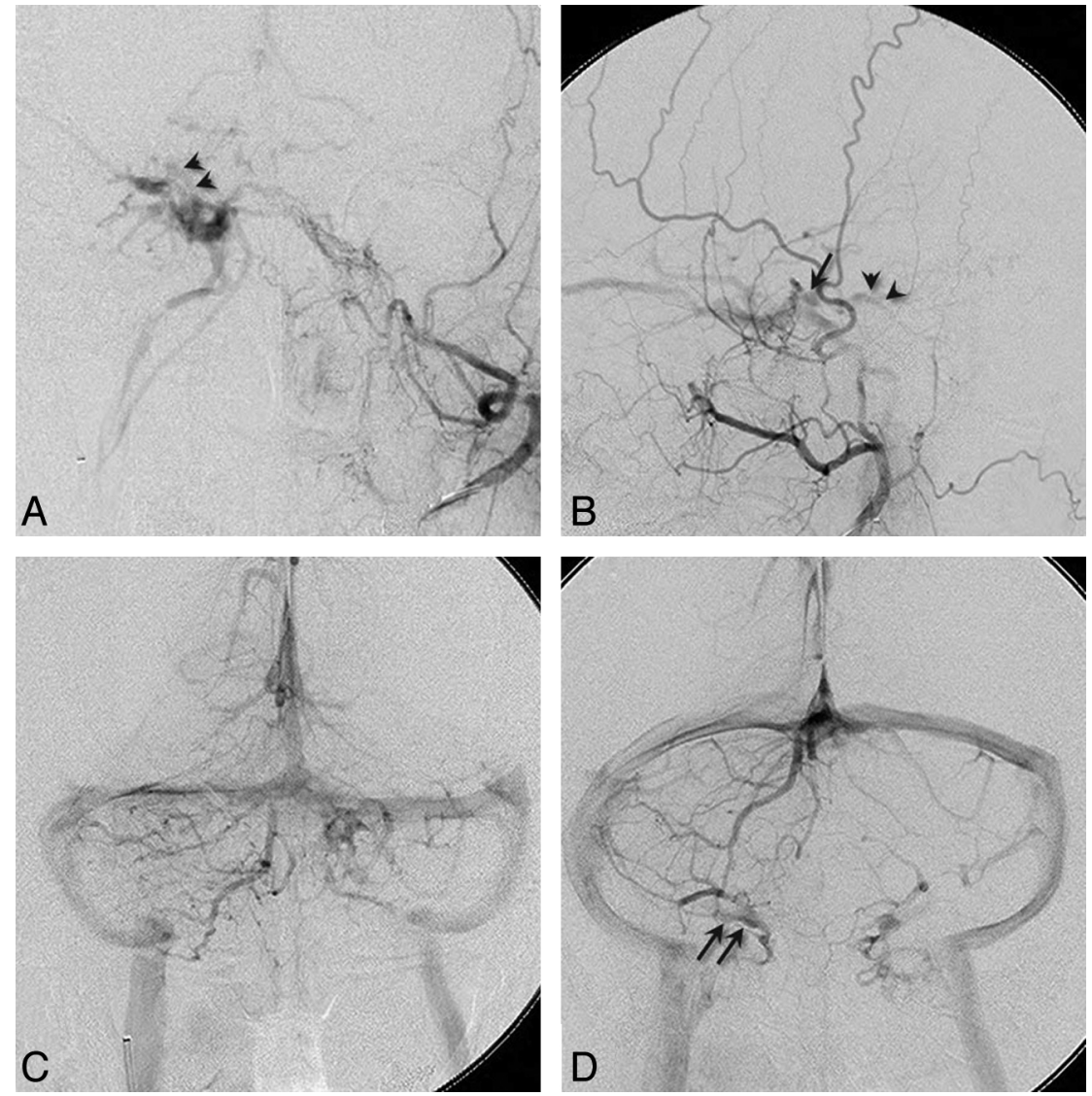

FIG 8. CSDAVF with SPS drainage. Frontal $(A)$ and lateral $(B)$ views of the left internal maxillary angiogram show the AVF draining into the SPS (arrow) and the petrosal vein (arrowheads). Other drainage routes of the superior ophthalmic vein, the superficial middle cerebral vein, and the inferior petrosal sinus are also noted. C. Frontal view of the right vertebral angiogram shows no visualization of the right SPS and the obvious venous congestion of the right cerebellar hemisphere. $D$, Frontal view of the right vertebral angiogram after transvenous embolization shows normal venous drainage of the right SPS on the cavernous sinus side (arrows). 

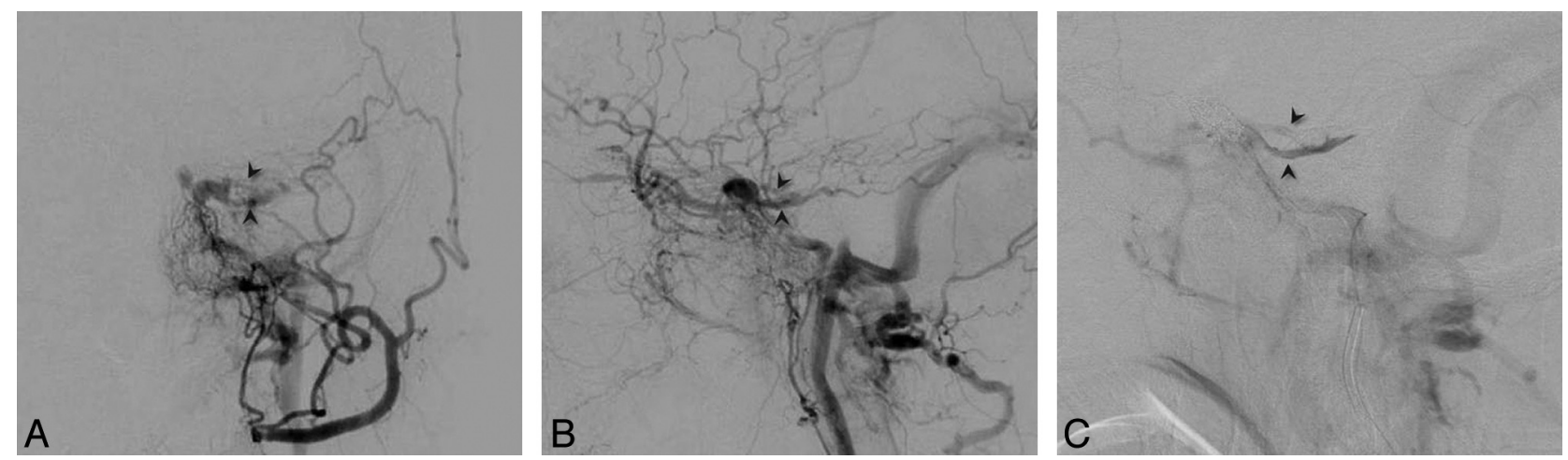

FIG 9. The duplication of the SPS in CSDAVF. Frontal $(A)$ and lateral $(B)$ views of the left internal maxillary angiogram show the CSDAVF draining into the left duplicated SPS (arrowheads). Additional drainage routes of the superior ophthalmic vein, the inferior petrosal sinus, and the superficial middle cerebral vein are also noted. C, Selective venography by the inserted microcatheter into the SPS shows the duplication of the SPS (arrowheads).

prenatal stage, the tentorial sinus extends medially and anastomoses with the cavernous sinus and pericavernous venous plexuses or sinuses. Different explanations have been presented regarding the development of the SPS. In 1957, Butler ${ }^{6}$ demonstrated that the anastomotic vein around the trigeminal nerve (peritrigeminal vein) and the anterior remnant of prootic sinus form the SPS. This explanation, however, does not mention the pial venous drainage. Padget ${ }^{7}$ proposed that the posterior part of the SPS first develops as a drainage vein of the mesencephalic vein (the petrosal vein). Later, the dorsal remnant of the primitive tentorial sinus anastomoses between the cavernous sinus and the SPS at the entrance of the petrosal vein, which then becomes the anterior segment of the SPS. This anastomosis is sometimes plexiform or poorly developed.

According to the explanation of the development of the SPS by Padget, ${ }^{7}$ the SPS develops primarily as a drainage route from the petrosal vein, receiving blood from the anterior cerebellar and brain stem venous systems. This corresponds well to our results regarding the hemodynamic feature of the SPS. In our results, demonstration of the SPS on carotid angiography is highly associated with the variation of cerebral venous drainage, including hypogenesis of the basal vein of Rosenthal and the sphenopetrosal type of the superficial middle cerebral vein (persistent primitive tentorial sinus), which also supports Padget's explanation. On VAG, approximately $50 \%$ of the SPS demonstrated partially, at either the anterior segment or the posterior segment, to receive blood from the petrosal vein. Disconnection of the anterior and posterior segment of the SPS was also seen in a few patients. These findings would represent hypoplasty or malfusion of the anterior and posterior segments of the SPS in development. Duplication of the SPS would be due to the plexiform feature of the remnant of the primitive tentorial sinus.

\section{Relationship of the Types of SPS with SPS Drainage of CSDAVFs}

In our results, SPS drainage from CSDAVFs was found in $7 \mathrm{pa}-$ tients (28\% of all 25 patients with CSDAVF and $37 \%$ of 16 patients with inferior petrosal sinus occlusion). Among the 7 patients with SPS drainage, CSDAVFs drained through the whole
SPS from the cavernous sinus in 3 patients. In the other 3 patients, DAVFs drained through the anterior segment of the SPS into the tributes of the petrosal vein without draining into the posterior segment of the SPS. Although stenosis of the dural sinus may develop secondary to high-flow angiopathy caused by the arteriovenous fistulas, SPS drainage and its drainage patterns would be associated with variation of anatomy of the SPS. When CSDAVFs occur in patients with hypoplasty of the posterior segment of the SPS, the fistulas have a higher risk of venous reflux into the cerebellopontine veins compared with the case in which the whole SPS is present. Regarding transvenous embolization, an approach via the SPS is applied in some patients. ${ }^{8}$ These variations of segmental hypoplasty and plexiform features of the SPS could affect accessibility via the SPS. Furthermore, multiplication of the SPS may have a risk of residual SPS drainage after transvenous embolization.

\section{CONCLUSIONS}

Our study suggests that the SPS normally works as a drainage route receiving blood from the anterior cerebellar and brain stem venous systems and does not work as a normal drainage route from the cavernous sinus. More than half of the SPSs have segmentally hypoplastic features on angiography. The variation of the hemodynamic features would be related to the relatively lower frequency and to the 2 different types of SPS drainage from CSDAVFs.

\section{REFERENCES}

1. Wetzel SG. Cerebral arteries and veins. In: Rubin DG, Rofsky NM, eds. CT and MR Angiography: Comprehensive Vascular Assessment. Philadelphia: Lippincott Williams \& Wilkins; 2009:381-449

2. Anzalone N, Tartaro A. Intracranial MR angiography. In: Schneider G, Prince MR, Meaney JF, et al, eds. Magnetic Resonance Angiography: Techniques, Indications, and Practical Application. Milan, Italy: Springer-Verlag Italia; 2005:103-40

3. Stiebel-Kalish H, Setton A, Nimii Y, et al. Cavernous sinus dural arteriovenous malformations: patterns of venous drainage are 
related to clinical signs and symptoms. Ophthalmology 2002;109: 1685-91

4. Kiyosue $\mathrm{H}$, Mori $\mathrm{H}$, Sagara $\mathrm{Y}$, et al. Basal cerebral venous drainage from cavernous sinus dural arteriovenous fistulas. Neuroradiology 2009;51:175-81

5. Suh DC, Lee JH, Kim SJ, et al. New concept in cavernous sinus dural arteriovenous fistula: correlation with presenting symptom and venous drainage patterns. Stroke 2005;36:1134-39
6. Butler $\mathrm{H}$. The development of certain human dural venous sinuses. J Anat 1957;91:510-26

7. Padget $\mathrm{DH}$. The development of the cranial venous system in man, from the viewpoint of comparative anatomy. Contrib Embryol 1957;36:79-140

8. Mounayer C, Piotin M, Spelle L, et al. Superior petrosal sinus catheterization for transvenous embolization of a dural carotid cavernous sinus fistula. AJNR Am J Neuroradiol 2002;23:1153-55 